\title{
Tuberculosis contact investigation following the stone- in-the-pond principle in the Netherlands - Did adjusted guidelines improve efficiency?
}

Sarah van de Berg ${ }^{1}$, Connie Erkens ${ }^{1}$, Christiaan Mulder $^{1,2}$

1. KNCV Tuberculosis Foundation, The Hague, the Netherlands

2. Amsterdam Institute for Global Health and Development, Amsterdam University Medical Center, Amsterdam, the Netherlands

Correspondence: Christiaan Mulder (christiaan.mulder@kncvtbc.org)

Citation style for this article:

van de Berg Sarah, Erkens Connie, Mulder Christiaan. Tuberculosis contact investigation following the stone-in-the-pond principle in the Netherlands - Did adjusted guidelines improve efficiency?. Euro Surveill. 2021;26(45):pii=2001828. https://doi.org/10.2807/1560-7917. ES.2021.26.45.2001828

Background: In low tuberculosis (TB) incidence countries, contact investigation $(\mathrm{Cl})$ requires not missing contacts with TB infection or disease without unnecessarily evaluating non-infected contacts. Aim: We assessed whether updated guidelines for the stonein-the-pond principle and their promotion improved $\mathrm{Cl}$ practices. Methods: This retrospective study used surveillance data to compare $\mathrm{Cl}$ outcomes before (20112013) and after (2014-2016) the guideline update and promotion. Using negative binomial regression and logistic regression models, we compared the number of contacts invited for $\mathrm{Cl}$ per index patient, the number of $\mathrm{Cl}$ scaled-up according to the stone-in-the-pond principle, the TB and latent TB infection (LTBI) testing coverage, and yield. Results: Pre and post update, 1,703 and 1,489 index patients were reported, 27,187 and 21,056 contacts were eligible for $\mathrm{Cl}, 86 \%$ and $89 \%$ were tested for TB, and $0.70 \%$ and $0.73 \%$ were identified with active TB, respectively. Post update, the number of casual contacts invited per index patient decreased statistically significantly $(\mathrm{RR}=0.88 ; 95 \% \mathrm{Cl}$ : $0.79-0.98)$, TB testing coverage increased $(O R=1.4$; $95 \% \mathrm{Cl}: 1.2-1.7)$, and $\mathrm{TB}$ yield increased $(\mathrm{OR}=2.0$; $95 \% \mathrm{Cl}: 1.0-3.9)$. The total LTBI yield increased from $8.8 \%$ to $9.8 \%$, with statistically significant increases for casual $(\mathrm{OR}=1.2 ; 95 \% \mathrm{Cl}: 1.0-1.5)$ and community contacts $(\mathrm{OR}=2.0 ; 95 \% \mathrm{Cl}: 1.6-3.2)$. The proportion of Cls appropriately scaled-up to community contacts increased statistically significantly $(R R=1.8 ; 95 \%$ $\mathrm{Cl}$ : 1.3-2.6). Conclusion: This study shows that promoting evidence-based $\mathrm{Cl}$ guidelines strengthen the efficiency of Cls without jeopardising effectiveness. These findings support $\mathrm{Cl}$ is an effective TB elimination intervention.

\section{Introduction}

The Netherlands is a low tuberculosis (TB) incidence country with 4.7 new TB patients per 100,000 population; in 2018, 806 patients were notified [1]. Since the 1980 s, contact investigation $(\mathrm{Cl})$ has been one of the pillars of TB control and is considered essential for the prevention of outbreaks and transmission [2,3]. In high burden and low resource settings, $\mathrm{Cl}$ focusses primarily on TB screening of people living with HIV (PLHIV), children younger than 5 years old [4], and household and close contacts of index patients with sputum smear-positive pulmonary TB or drug-resistant TB (DRTB). In low burden and high resource settings such as the Netherlands, $\mathrm{Cl}$ takes on a broader focus, which includes identifying other exposed contacts, contacts of sputum smear-negative patients and the transmission source of TB patients who are likely to have been recently infected (source or reverse $\mathrm{Cl}$ ) $[3,5]$. The Dutch guidelines recommend conducting a $\mathrm{Cl}$ of potentially infectious TB patients [6], i.e., patients with cultureconfirmed pulmonary TB and with extrapulmonary TB where transmission may have occurred. Source investigation should be considered for all recently infected TB patients if the source patient is unknown or not diagnosed and likely traceable in the Netherlands.

$\mathrm{Cl}$ should use a sequence of priority decisions to identify all contacts with active TB or latent TB infection (LTBI) without screening non-exposed or low risk contacts as such efforts would be an inappropriate use of public resources [2]. In the Netherlands, $\mathrm{Cl}$ is conducted according to the stone-in-the-pond principle: contacts are prioritised for testing in concentric circles around the index patient, depending on the level of exposure and vulnerability of the contact, until the prevalence of infection approximates that of the local community [7].

Since $2006, \mathrm{Cl}$ results have been recorded in the national TB surveillance registry. This registry allows for the monitoring and evaluation of national policy and the performance of the Public Health Services (PHSs) responsible for conducting the $\mathrm{Cl}$. Evaluation of the data between 2006 and 2010 showed that $\mathrm{Cl}$ had an active TB yield of $0.4 \%$ and a LTBI yield of $5 \%$ [8]. During this period, however, the testing coverage for 


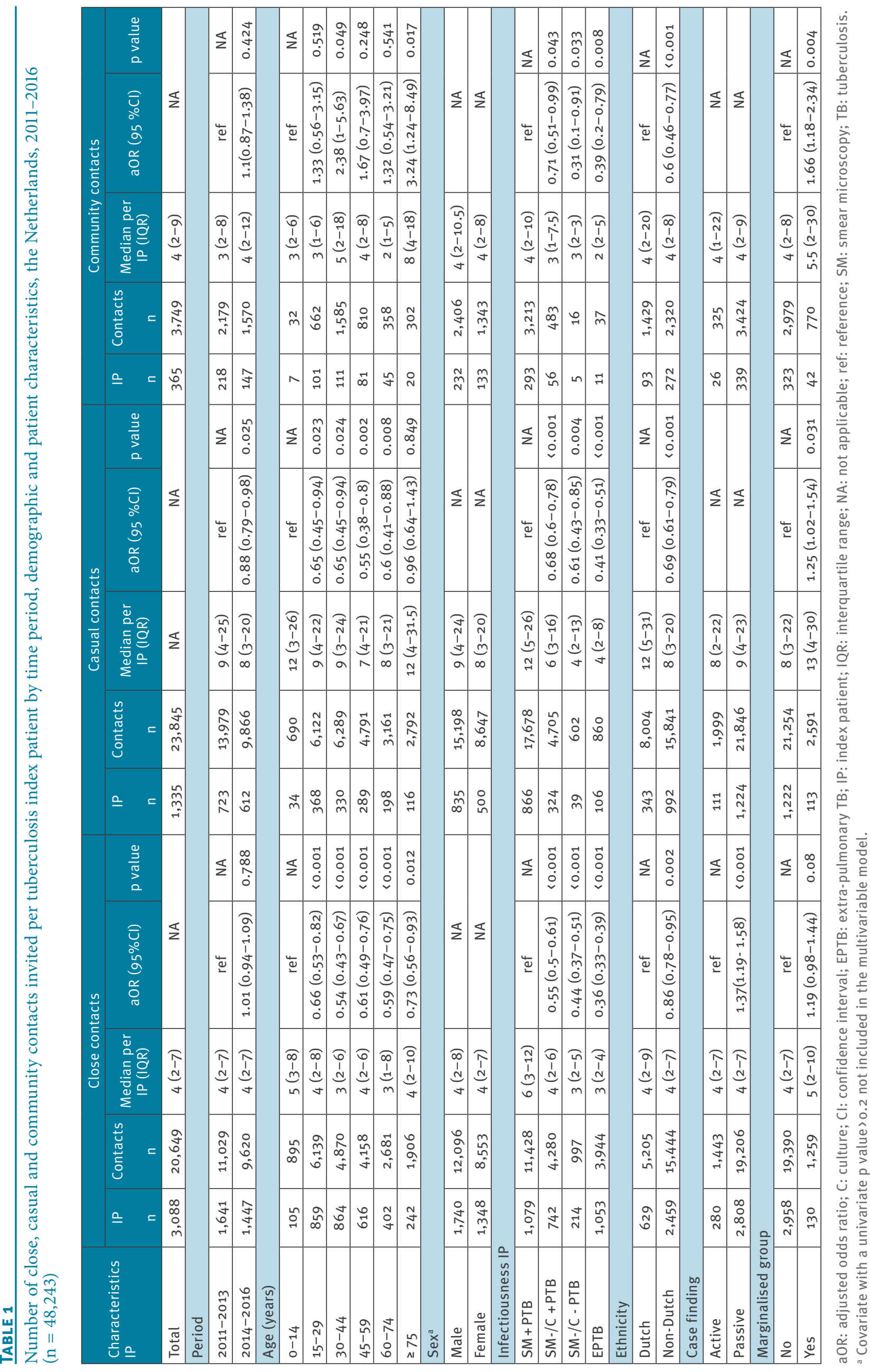




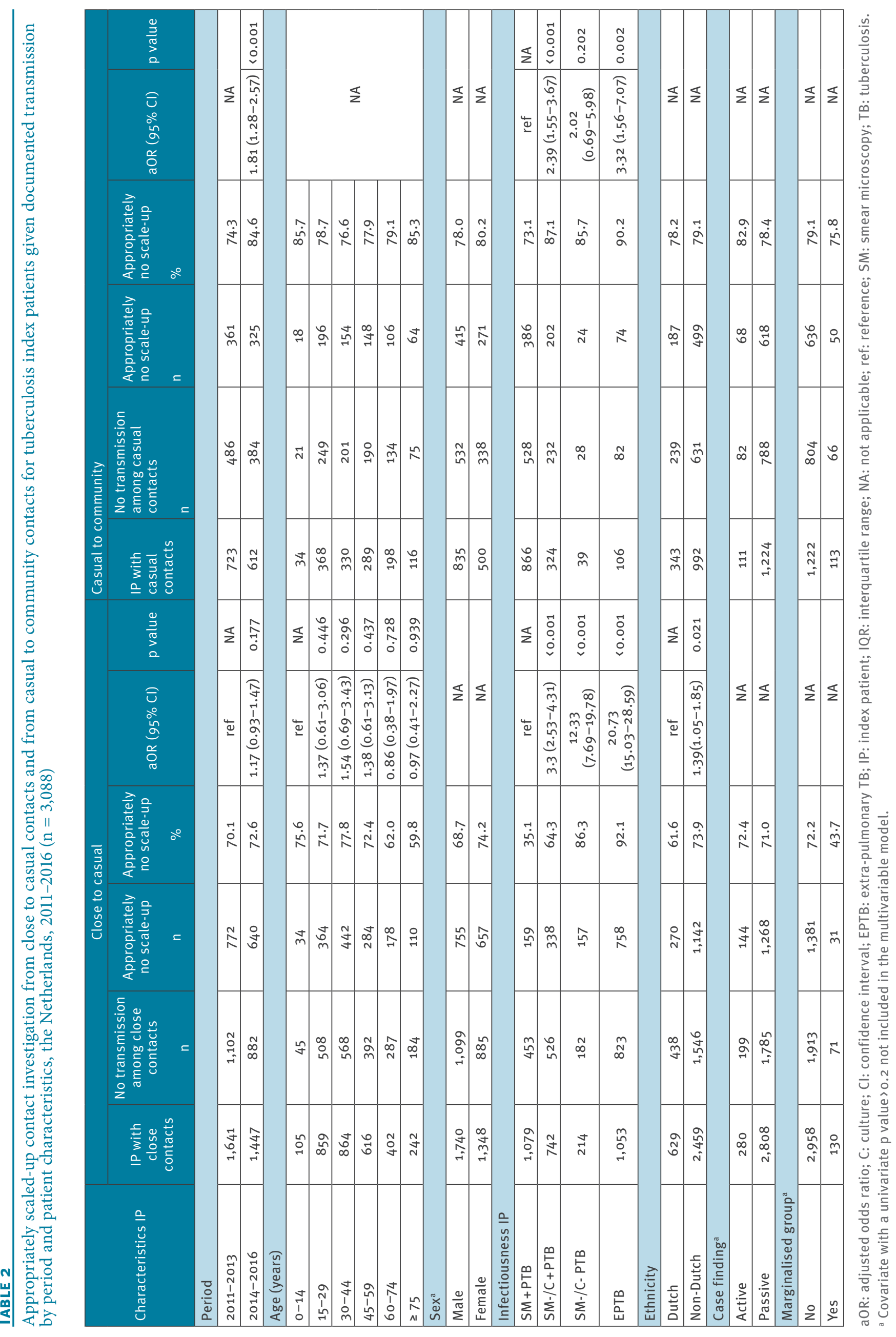


LTBI was low (73\%) as BCG-vaccinated contacts and contacts from high burden countries were not eligible for LTBI testing until 2010, when interferon gamma release assays (IGRAs) were recommended for use in these populations. Qualitative research showed that the national guidelines were not followed completely, and public health nurses did not fully adhere to the stone-in-the pond principle [9]. Based on these findings, it was deemed likely that the TB and LTBI yields could be increased by improving the targeting of individuals eligible for $\mathrm{Cl}$ through the stone-in-thepond principle and by providing LTBI testing for BCGvaccinated contacts and contacts from high burden countries. $\mathrm{Cl}$ guidelines [6] were updated accordingly in 2013 [8]. Dissemination and implementation of the guideline changes were supported through the development of operational guidance and tools as well as nation-wide 2 -day multidisciplinary on-site trainings of all healthcare staff of the PHSs involved in $\mathrm{Cl}$ of TB. The training is mandatory for TB nurses and physicians working at the PHSs and is offered on an annual basis to all new professionals.

The objective of this study was to determine whether the guideline adaptation in 2013 resulted in more efficient but equally or more effective $\mathrm{Cl}$ practices by determining whether there was a decrease in the number of contacts being invited for $\mathrm{Cl}$ per index patient, an increase in the number of $\mathrm{Cl}$ scaled-up according to the stone-in-the-pond principle, and an increase in TB and LTBI testing coverage while the relative yield of active TB and LTBI remained similar or increased.

\section{Methods}

This retrospective cohort study used records of TB patients registered in the Netherlands Tuberculosis Register (NTR) between 1 January 2011 and 31 December 2016. Records were included if a $\mathrm{Cl}$ was initiated. Patients with incomplete or inconclusive $\mathrm{Cl}$ data were excluded. If a $\mathrm{Cl}$ had more than 200 invited contacts, it was considered an outlier and therefore excluded. The efficiency and effectiveness were compared between $\mathrm{Cl}$ of patients registered between 1 January 2011 and 31 December 2013 ('before the guideline adaptation') and $\mathrm{Cl}$ of patients registered between 1 January 2014 and 31 December 2016 ('after the guideline adaptation'). We compared the number of contacts invited for $\mathrm{Cl}$ per index patient, the number of $\mathrm{Cl}$ scaled-up according to the stone-in-the-pond principle, the TB and LTBI testing coverage, as well as the relative yield of active TB and LTBI.

According to national guidelines, the first priority group of contacts include those considered most exposed to the index patient (household contacts and other close contacts) as well as vulnerable contacts (children younger than 5 years old and immunocompromised persons). Priority contacts are determined by PHS staff based on information collected from the index patient during a personal interview [6] (Supplement S1). Proof of transmission is defined as a contact identified with active TB, a child younger than 5 years old with LTBI, or prevalence of LTBI among evaluated contacts at least twice as high than the expected background LTBI prevalence based on country of origin and age (Supplementary Table S2). When the number of identified close contacts is too small to properly examine transmission, it is common practice to include a subgroup of casual contacts who are considered second most exposed to the index patient [6]. The screening algorithm for identified contacts is presented in Supplementary Table S3.

The coverage of contacts tested for TB was defined as the number of contacts tested for TB divided by the number of contacts invited for $\mathrm{Cl}$. The coverage of contacts tested for LTBI was defined as the number of contacts tested for LTBI (tuberculin skin test (TST) and/or an IGRA) divided by the number of contacts invited for $\mathrm{Cl}$. The yield of TB and LTBI was defined as the number of contacts identified with TB and LTBI, respectively, divided by the number of contacts tested for TB and LTBI, respectively. LTBI was defined as being TST and/ or IGRA positive according to the national guidelines. Because the NTR data are aggregated per index patient, individual contact-based data were not available.

\section{Statistical analysis}

The number of contacts invited per index patient before and after the guideline adaptation were compared using negative binomial regression. The number of $\mathrm{Cl}$ where the stone-in-the-pond principle was appropriately applied was compared using logistic regression. To correct for the effect of the number of close contacts on the appropriate scale-up to casual contacts, the number of close contacts investigated per $\mathrm{Cl}$ was included in this model as covariate.

TB and LTBI coverage and yield in the two periods were compared using generalised estimating equations (GEE) logistic regression model. We treated each index patient as a cluster as the NTR reports the number of contacts tested for TB/LTBI and identified with TB/LTBI aggregated per index patient. Models on number of contacts invited per index patient, coverage and yield were a priori stratified by priority of contact.

The following characteristics of the index patients were included as covariates and assessed for all models: sex; age $(0-14 ; 15-29 ; 30-44 ; 45-59 ; 60-74$; $75+$ ); infectiousness (smear-positive pulmonary TB, smear-negative, culture-positive pulmonary TB, smearnegative, culture-negative pulmonary TB and extrapulmonary TB); ethnicity (Dutch or non-Dutch); belonging to a marginalised group (individuals who are homeless, addicted to drugs or addicted to alcohol); and reason for examination - active (i.e., identified through screening) or passive (i.e., identified in clinical care through presentation of symptoms).

Covariates with a univariate $p$ value $\leq 0.2$ were included in the multivariable models. Subsequently, the most 


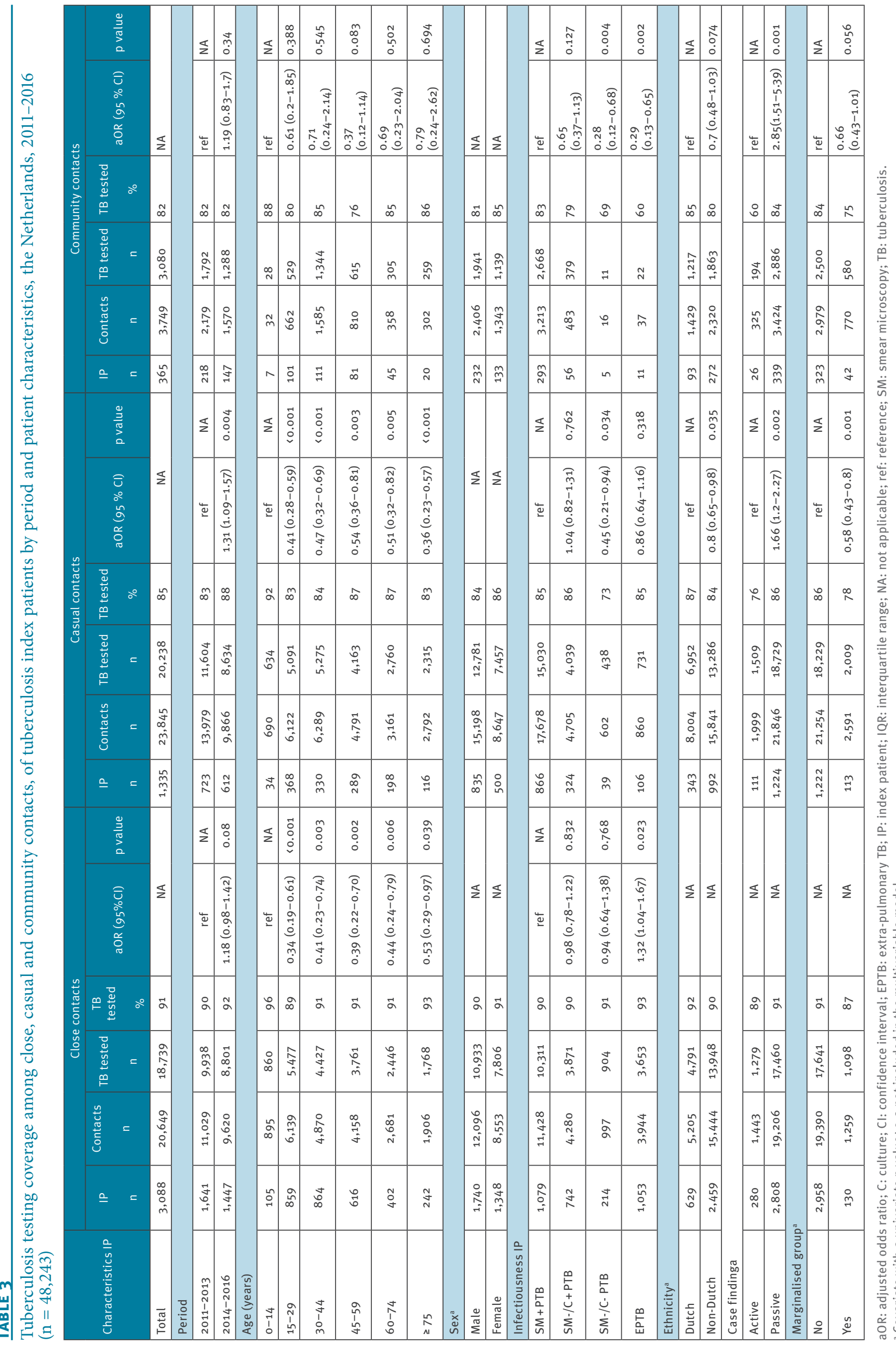


parsimonious model was selected by backward elimination guided by the change and coefficients and log likelihood, if applicable, of successive models. A p value $\leq 0.05$ was regarded as statistically significant. All statistical analysis were performed in SPSS version 25.0 (SPSS, Chicago, IL, United States).

\section{Ethical statement}

The NTR Registration Commission approved the use of the NTR data. Ethical approval was not required as the data were anonymised and aggregated retrospective surveillance data.

\section{Results}

Between 2011 and 2016, 5,368 patients were registered in the NTR. After cleaning the data and applying the selection criteria, 3,192 index patients were included in the analyses $-1,703$ before and 1,489 after the guideline adaptation (Supplementary Figure $\mathrm{S}_{4}$ ). Of all the Cls, $0.5 \%(n=8)$ before and $0.6 \%(n=11)$ after guideline adaptation included more than 200 contacts and because these were outliers, they were excluded from further analysis. Of the 3,192 index patients, 3,088, 1,335 , and 365 had close, casual and community contacts eligible for $\mathrm{Cl}$, respectively. The characteristics of the index patients were comparable in both periods: about 35\% had smear positive PTB, about 90\% were passively identified and about $80 \%$ were of non-Dutch origin (Supplementary Table $\mathrm{S}_{5}$ ).

\section{Number of contacts invited per contact investigation}

Before the guideline adaptation, 27,187 (median 6 per $\mathrm{Cl}$; IQR: 3-18) contacts were identified for $\mathrm{Cl}$; after the guideline adaptation, 21,056 (median 6 per $\mathrm{Cl}$; IQR: 3-15) were identified for $\mathrm{Cl}$. The number of casual contacts invited per $\mathrm{Cl}$ decreased statistically significant from a median of 9 (IQR: 4-25) to a median of 8 (IQR: 3-20) $(\mathrm{RR}=0.88 ; 95 \% \mathrm{Cl}: 0.79-0.98 ; \mathrm{p}=0.025)$ (Table 1). There was no decrease in the number of close and community contacts invited (Table 1 ). In all close, casual and community contacts, the number of contacts invited per $\mathrm{Cl}$ was (marginally) larger for smear-positive index patients, Dutch index patients and patients belonging to a marginalised group (Table 1). For close and casual contacts, the number of contacts invited per $\mathrm{Cl}$ was also larger for index patients younger than 15 years old (Table 1 ). For close contacts, the number of contacts invited per $\mathrm{Cl}$ was larger for passively identified index patients (Table 1 ).

\section{Appropriate scale-up}

The proportion of $\mathrm{Cls}$ appropriately not scaled-up to casual contacts given the absence of evidence for transmission among close contacts increased from $70.1 \%(772 / 1,102)$ between 2011 and 2013 to $72.6 \%$ (640/882) between 2014 and 2016. This increase was not statistically significant $(R R=1.17 ; 95 \% \mathrm{Cl}$ : 0.93-1.47; $p=0.177$ ) (Table 2). The proportion of Cls appropriately not scaled-up to community contacts increased statistically significantly, from $74.3 \%$
(361/486) between 2011 and 2013 to $84.6 \%$ (235/384) between 2014 and $2016(\mathrm{RR}=1.81$; $95 \% \mathrm{Cl}: 1.28-2.57$; $\mathrm{p}=0.001$ ) (Table 2). Appropriate scaling up from close to casual contacts was independently associated with smear negative pulmonary or extrapulmonary TB disease and non-Dutch ethnicity, and appropriate scaling up from casual to community contacts was independently associated with smear negative pulmonary or extrapulmonary TB disease (Table 2).

\section{Tuberculosis testing coverage}

The overall TB testing coverage increased from $85.8 \%$ $(23,334 / 27,187)$ between 2011 and 2013 to $88.9 \%$ $(18,723 / 21,056)$ between 2014 and 2016. The testing coverage increased statistically significantly among casual contacts, from $83 \%$ to $88 \%(\mathrm{OR}=1.43 ; 95 \%$ $\mathrm{Cl}$ : 1.18-1.74; $\mathrm{p}<0.001$ ) (Table 3). The testing coverage increased borderline statistically significantly for close contacts, from $90 \%$ to $92 \%(\mathrm{OR}=1.18 ; 95 \% \mathrm{Cl}$ : $0.98-1.42 ; p=0.08$ ) (Table 3 ). The testing coverage did not change for community contacts (Table 3). For close and casual contact, the testing coverage was higher among contacts of index patients younger than 15 years old. For casual contacts, the testing coverage was higher for contacts of index patients of Dutch origin and passively detected index patients but not for socially marginalised risk groups (Table 3 ). For community contacts, coverage of TB testing was higher among contacts of index patients detected passively (Table 3 ).

\section{Latent tuberculosis infection testing coverage}

The overall LTBI testing coverage increased from $73 \%(19,964 / 27,187)$ between 2011 and 2013 to $85 \%$ $(17,843 / 21,056)$ between 2014 and 2016. The LTBI testing coverage increased statistically significantly among close contacts $(75.7 \%$ vs $86.0 \% ; O R=2.0 ; 95 \% \mathrm{Cl}$ : $1.7-2.4 ; p<0.001)$, casual contacts (72.4\% vs $84.2 \%)$ $(\mathrm{OR}=1.995 \% \mathrm{Cl}: 1.7-2.3 ; \mathrm{p}<0.001)$ and community contacts $(69.0 \%$ vs $80.6 \%)(\mathrm{OR}=2.2 ; 95 \% \mathrm{Cl}: 1.5-3.0$; $\mathrm{p}<0.001$ ) (Table 4). In all three groups, the coverage of LTBI testing was statistically significantly higher among contacts of index patients younger than 15 years old (Table 4). For close and casual contacts, the coverage of LTBI testing was statistically significantly higher among contacts of Dutch index patients and index patients not belonging to a socially marginalised group (Table 4). For close and community contacts, the coverage of LTBI testing was statistically significantly higher among contacts of index patients with sputum positive pulmonary TB (Table 4).

\section{Tuberculosis yield}

The yield of active TB among contacts increased from $0.70 \%(164 / 23,334)$ to $0.73 \%(136 / 18,723)$ after guideline adaptation. The TB yield increased statistically significantly among casual contacts, from $0.17 \%$ to $0.28 \%(\mathrm{OR}=2.0 ; 95 \% \mathrm{Cl}: 1.0-3.9 ; \mathrm{p}=0.045)$ (Table 5). There was no statistically significant difference in the TB yield among close contacts (1.4\% vs $1.3 \%$ ) $(\mathrm{OR}=0.97 ; 95 \% \mathrm{Cl}: 0.68-1.4 ; \mathrm{p}=0.854)$ (Table 6). The yield among community contacts (0.11\% vs o\%) could 


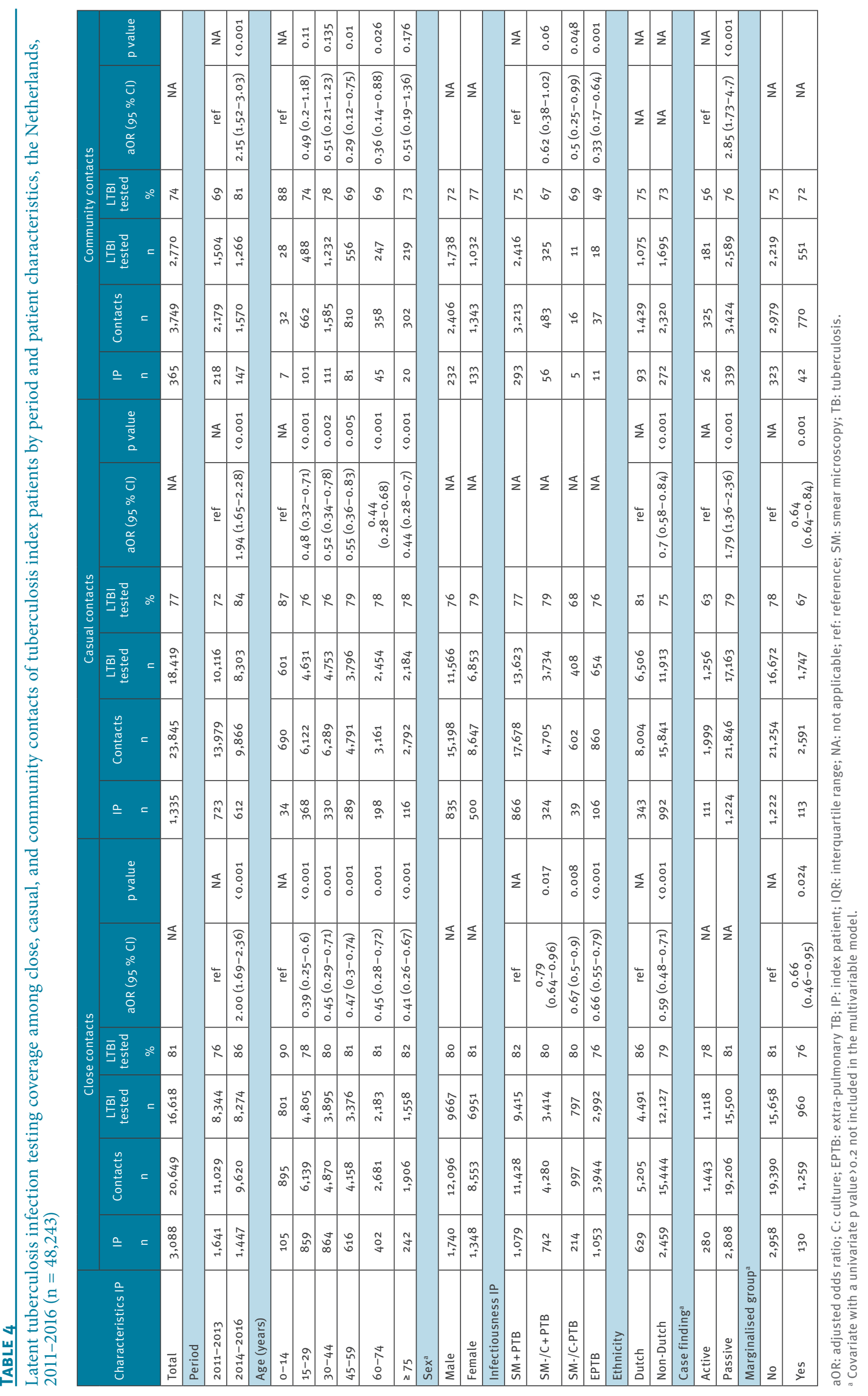




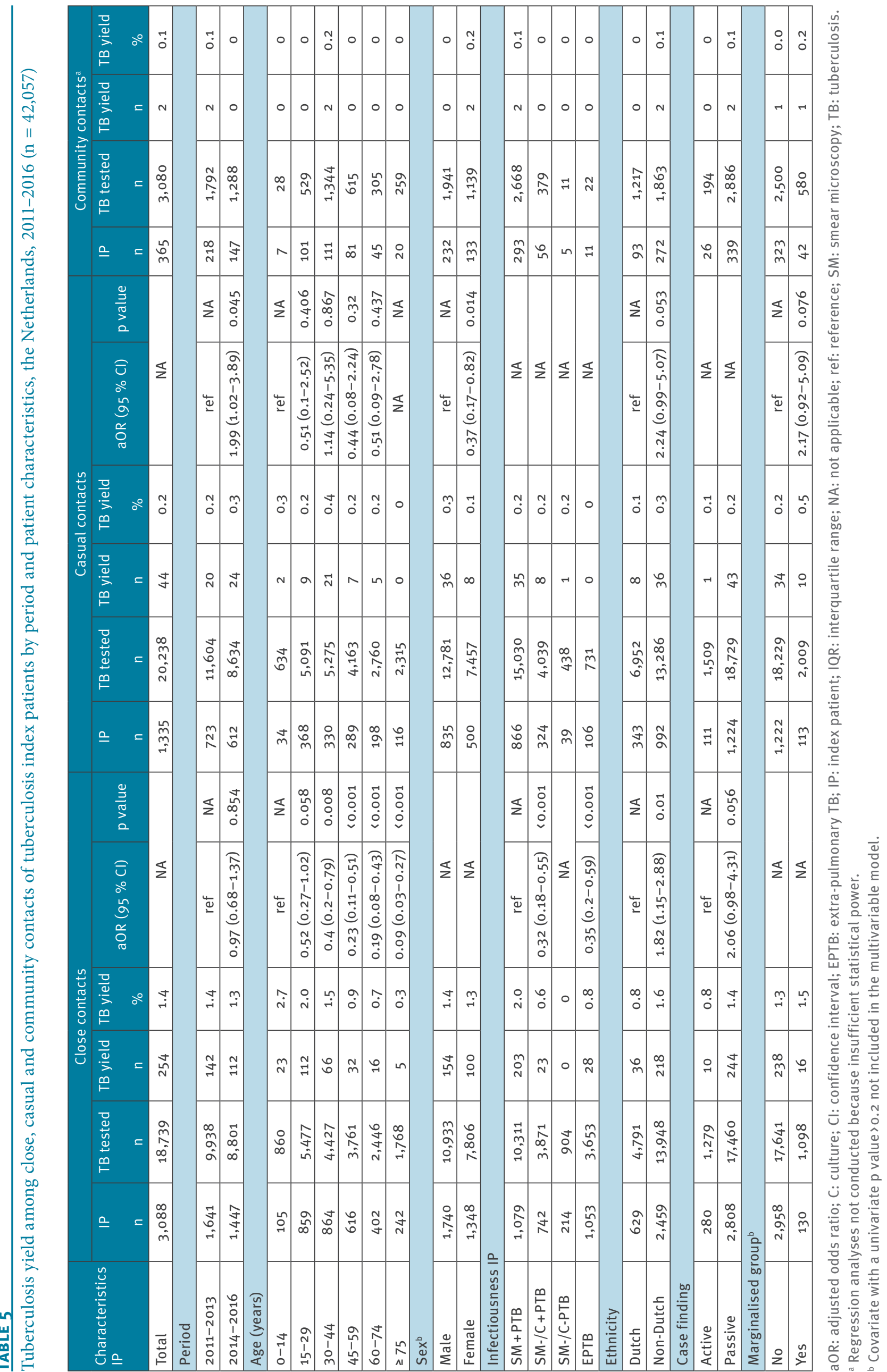




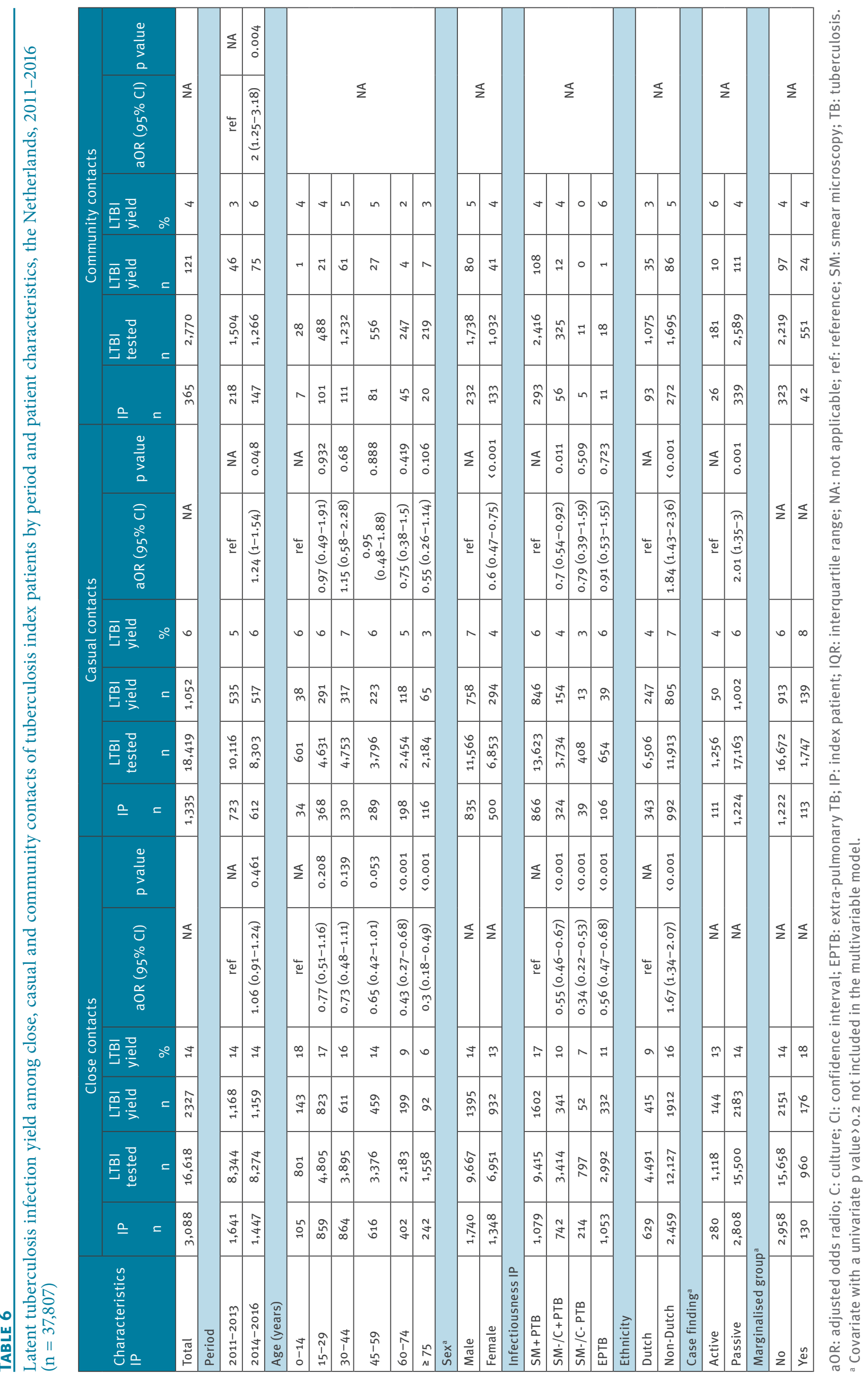


not be compared statistically as too few patients $(n=2)$ were identified among this group. In the stratified analysis per contact group, characteristics of the index patient independently associated with a higher yield of TB diagnosis among close contacts were age $<30$ years, sputum positive pulmonary TB and non-Dutch ethnicity (Table 5). For casual contacts, male sex was the only characteristic of the index patient associated with a higher yield of contact investigation, and non-Dutch ethnicity was borderline statistically significant (Table 5).

\section{Latent tuberculosis infection yield}

The yield of LTBI among contacts increased from $8.8 \%(1,749 / 19,964)$ between 2011 and 2013 to $9.8 \%$ $(1,751 / 17,843)$ between 2014 and 2016 . The yield of LTBI increased statistically significantly for casual ( $5.3 \%$ vs $6.2 \%)(\mathrm{OR}=1.2 ; 95 \% \mathrm{Cl}: 1.0-1.5 ; \mathrm{p}=0.048)$ (Table 6) as well as community contacts ( 3.1 vs $5.9 \%)(O R=2.0$; 95\% Cl: $1.6-3.2 ; p=0.004$ ) (Table 6). There was no statistically significant difference in the LTBI yield among close contacts (Table 6). In the stratified analysis per contact group, characteristics of the index patient independently associated with a higher yield for LTBI diagnosis among close contacts were age<60 years, sputum positive pulmonary disease and non-Dutch ethnicity (Table 6). For casual contacts, independently associated characteristics were female sex, non-Dutch ethnicity and passive case finding. Smear-negative, culture-positive pulmonary TB was negatively associated with a higher LTBI yield (Table 6).

\section{Discussion}

In this study, we showed that adapting $\mathrm{Cl}$ guidelines with a stronger focus on the stone-in-the-pond principle and clear dissemination and training efforts may have resulted in more efficient $\mathrm{Cl}$ and increased the overall relative TB and $\mathrm{LTB}$ yield among contacts. The TB yield among close contacts (1.4\%) did not change significantly and is comparable to other low burden, high-income countries [10-12]. The yield of TB among casual contacts of $0.4 \%(0.2-0.6 \%)$ increased statistically significantly and became comparable to the TB yield among causal contacts in other high-income countries [10].

Cls were more often appropriately scaled up from casual to community contacts, indicating an improved risk assessment of the TB contacts and stricter adherence to the stone-in-the-pond principle as recommended in the updated guidelines. As fewer contacts were screened, the relative TB yield increased.

To the authors' knowledge, no studies have evaluated the yield of $\mathrm{Cl}$ among community contacts. WHO guidelines do not recommend extending $\mathrm{Cl}$ to community contacts [4]. However, for low burden countries, it is recommended to screen for $\mathrm{LTBI}$ and treat risk groups that have a high likelihood of recent TB transmission [3]. US guidelines state that 'low-priority contacts' may be included if resources permit and the programme meets its performance goals [13]. The United Kingdom (UK) guidelines apply the stone-in-the-pond principle but do not differentiate between casual and community contacts [14]. Between 2011 and 2016, two community contacts were identified with TB (60 per 100,000 community contacts investigated). Despite a low numeric yield, the identification of community contacts eligible for $\mathrm{Cl}$ is compliant with the national criteria for a target group of active case findings for TB, which is defined as a population with a prevalence or annual incidence of 50 TB patients per 100,000 persons.

The relative yield of LTBI among casual and community contacts screened for LTBI was higher after guideline adaption. This increase possibly resulted from better LTBI testing coverage, which improves decision making about whether to scale up to the next priority group. This improved prioritisation may explain the increase in the median number of community contacts invited (from 3 to 4 contacts) although this was not statistically significant. The LTBI yield among close and casual contacts, however, remained lower compared with other high-income countries $[15,16]$. This difference may result from variations in background prevalence and $\mathrm{Cl}$ policies regarding contact eligibility, enrolment and diagnostic tests.

Significantly more contacts of foreign-born TB patients were offered and accepted LTBI testing. This may contribute substantially to eliminating TB in the Netherlands. The number of foreign-born persons with LTBI notified to the NTR and identified through CI increased by $21 \%$ in the period 2014 to 2016 compared with 2011 to 2013 [17-21], and the number of Dutch-born TB contacts with LTBI decreased by $19 \%$. According to the national surveillance report from $2018,78 \%$ of the contacts identified with LTBI were provided tuberculosis preventive treatment (TPT); in 2017, $88 \%$ completed the treatment [1]. These percentages are in line with the European consensus on $\mathrm{Cl}$ target proportions for infected contacts on TPT initiation ( $85 \%$ ) and TPT completion (75\%) [22].

Our study has a few limitations. The classification of the contact group is determined by the public health nurse based on an assessment of the intensity and frequency of the contact with the index patient. As the NTR data cannot be used to verify classification, there may have been some over- or underestimation of the true number invited, coverage and yield per contact group. However, given the reduction of the median number of casual contacts before and after the trainings, it is likely that the recommendations for classification were followed more accurately.

The NTR does not provide any characteristics of the individual contacts as contact data are aggregated per index patient. Hence changes in contact populations before and after the guideline adaption could not be analysed, which may have biased the TB and LTBI yield. Overall, the surveillance data registered in the 
NTR may not reflect all improvements achieved through the guideline adaption and the corresponding training activities. However, surveillance data show significant positive trends in $\mathrm{Cl}$ outcomes and provide a basis for further investigations into $\mathrm{Cl}$ practices.

\section{Conclusion}

This study shows how the adherence to $\mathrm{Cl}$ guidelines based on the stone-in-the-pond principle can be monitored and evaluated. Careful implementation of new recommendations through nationwide training, administrative support and regular evaluation strengthens the efficiency of conducting Cls without jeopardising the yield. This is likely to improve the cost-effectiveness of $\mathrm{Cl}$.

\section{Conflict of interest}

None declared.

\section{Authors' contributions}

Conception and design: SvdB, CM, CE. Data collection and statistical analyses: SvdB. Interpretation and important intellectual input: all authors. First draft of manuscript: SvdB. Final version of the manuscript: all authors. All authors approved the final version submitted for publication.

\section{References}

1. Rijksinstituut voor Volksgezondheid en Milieu (RIVM). Tuberculose in Nederland 2018. [Tuberculosis in the Netherlands]. Bilthoven: RIVM. 2019. Dutch. Available from https://www.rivm.nl/bibliotheek/rapporten/2019-0188.pdf

2. Erkens CGM, Kamphorst M, Abubakar I, Bothamley GH, Chemtob D, Haas W, et al. Tuberculosis contact investigation in low prevalence countries: a European consensus. Eur Respir J. 2010;36(4):925-49. https://doi. org/10.1183/09031936.00201609 PMID: 20889463

3. Lönnroth K, Migliori GB, Abubakar I, D’Ambrosio $L$, de Vries G, Diel R, et al. Towards tuberculosis elimination: an action framework for low-incidence countries. Eur Respir J. 2015;45(4):928-52. https://doi. org/10.1183/09031936.00214014 PMID: 25792630

4. World Health Organization (WHO). Recommendations for investigating contacts of persons with infectious tuberculosis in low- and middle-income countries. Geneva: WHO. 2012. Available from: https://apps.who.int/iris/handle/10665/77741

5. Migliori GB, Zellweger JP, Abubakar I, Ibraim E, Caminero JA, De Vries G, et al. European Union standards for tuberculosis care. Eur Respir J. 2012;39(4):807-19. https://doi. org/10.1183/09031936.00203811 PMID: 22467723

6. Commissie voor Praktische Tuberculosebestrijding. KNCV Tuberculosefonds (KNCV Tuberculosis Foundation). Richtlijn - Tuberculose bron- en contactonderzoek. [Guidelines: Tuberculosis source and contact investigation]. The Hague: KNCV. 2013. Dutch.

7. Veen J. Microepidemics of tuberculosis: the stone-in-thepond principle. Tuber Lung Dis. 1992;73(2):73-6. https://doi. org/10.1016/0962-8479(92)90058-R PMID: 1643300

8. Tuberculosefonds KNCV. (KNCV Tuberculosis Foundation). Evaluatie bron- en contactonderzoek bij tuberculosepatiënten in Nederland, 2006-2010. [Evaluation of source and contact investigation of tuberculosis patients in the Netherlands, 2006-2010]. The Hague: KNCV. 2014. Dutch. Available from: https://www.kncvtbc.org/uploaded/2016/01/bco_ report 2006-2010.pdf

9. Mulder C, Harting J, Jansen N, Borgdorff MW, van Leth F. Adherence by Dutch public health nurses to the national guidelines for tuberculosis contact investigation. PLoS One. 2012;7(11):e49649. https://doi.org/10.1371/journal. pone.0049649 PMID: 23166738

10. Fox GJ, Barry SE, Britton WJ, Marks GB. Contact investigation for tuberculosis: a systematic review and meta-analysis. Eur Respir J. 2013;41(1):140-56. https://doi. org/10.1183/09031936.00070812 PMID: 22936710

11. Cavany SM, Sumner T, Vynnycky E, Flach C, White RG, Thomas $\mathrm{HL}$, et al. An evaluation of tuberculosis contact investigations against national standards. Thorax. 2017;72(8):736-45. https://doi.org/10.1136/thoraxjnl-2016-209677

12. Borraccino A, Migliore E, Piccioni P, Baussano I, Carosso A, Bugiani M. Yield of tuberculosis contact investigation in a lowincidence country. J Infect. 2014;68(5):448-54. https://doi. org/10.1016/j.jinf.2013.12.005 PMID: 24418415

13. National Tuberculosis Controllers AssociationCenters for Disease Control and Prevention (CDC). Guidelines for the Investigation of Contacts of Persons with Infectious Tuberculosis. Recommendations from the National Tuberculosis Controllers Association and CDC. MMWR Recomm Rep. 2005;54(RR-15):1-47.

14. National Institute for Health and Care Excellence (NICE). Tuberculosis Prevention, diagnosis, management and service organisation. NICE NG33 Methods, evidence and recommendations. London: NICE; Jan 2016. Available from: https://www.nice.org.uk/guidance/ng33/evidence/ full-guideline-80851860868

15. Moyo N, Tay EL, Denholm JT. Evaluation of tuberculin skin testing in tuberculosis contacts in Victoria, Australia, 20052013. Public Health Action. 2015;5(3):188-93. https://doi. org/10.5588/pha.15.0018 PMID: 26399290

16. Fox GJ, Barry SE, Britton WJ, Marks GB. Contact investigation for tuberculosis: a systematic review and meta-analysis. Eur Respir J. 2013;41(1). https://doi. org/10.1183/09031936.00070812

17. Rijksinstituut voor Volksgezondheid en Milieu (RIVM). Tuberculose in Nederland 2015. Surveillancerapport. [Tuberculosis in the Netherlands 2015. Surveillance report]. Bilthoven: RIVM. 2016. Dutch. Available from: https://www. rivm.nl/bibliotheek/rapporten/2016-0140.pdf

18. Tuberculosefonds KNCV. (KNCV Tuberculosis Foundation). Tuberculose in Nederland 2011. Surveillancerapport. [Tuberculosis in the Netherlands 2011. Surveillance report].The Hague: KNCV; Dec 2012. Dutch. Available from: https://www. kncvtbc.org/uploaded/2015/09/tin_2011_surveillancerapport. pdf

19. Rijksinstituut voor Volksgezondheid en Milieu (RIVM). Tuberculose in Nederland 2013. Surveillancerapport. [Tuberculosis in the Netherlands 2013. Surveillance report]. Bilthoven: RIVM; 2014. Dutch. Available from: https://www. rivm.nl/bibliotheek/rapporten/2014-0106.pdf

20. Rijksinstituut voor Volksgezondheid en Milieu (RIVM). Tuberculose in Nederland 2014. Surveillancerapport. [Tuberculosis in the Netherlands 2014. Surveillance report]. Bilthoven: RIVM; 2015. Dutch. Available from: https:// www.rivm.nl/sites/default/files/2018-11/TiN\%202014\%20 surveillancerapport.pdf

21. Rijksinstituut voor Volksgezondheid en Milieu (RIVM). Tuberculose in Nederland 2012. Surveillancerapport. [Tuberculosis in the Netherlands 2012. Surveillance report]. Bilthoven: RIVM; 2014. Dutch. Available from: https:// www.rivm.nl/sites/default/files/2018-11/TiN\%202012\%20 surveillancerapport\%20juni.pdf

22. Erkens CG, Kamphorst M, Abubakar I, Bothamley GH, Chemtob D, Haas W, et al. Tuberculosis contact investigation in low prevalence countries: a European consensus. Eur Respir J. 2010;36(4):925-49. https://doi. org/10.1183/09031936.00201609 PMID: 20889463

\section{License, supplementary material and copyright}

This is an open-access article distributed under the terms of the Creative Commons Attribution (CC BY 4.0) Licence. You may share and adapt the material, but must give appropriate credit to the source, provide a link to the licence and indicate if changes were made.

Any supplementary material referenced in the article can be found in the online version.

This article is copyright of the authors or their affiliated institutions, 2021. 